\title{
Highlights of this issue
}

\author{
BY SUKHWINDER S. SHERGILL
}

\section{POSTNATAL DEPRESSION AND MODIFYING DISRUPTIVE BEHAVIOUR}

Postnatal depression is likely to have an adverse effect on the mother-baby interaction. Poobalan and colleagues (pp. 378386) reviewed the literature to examine the effects on the children of mothers treated for postnatal depression. They report that maternal treatment was associated with improvement in mother-baby interactions, but also had beneficial effects on the cognitive development of the children in certain studies. Disruptive behaviour during childhood is predictive of maladjustment during adolescence and adulthood. Could a preventive intervention at this early stage act to reduce subsequent criminal behaviour and improve academic performance? Boisjoli et al (pp. 415-419) suggest that such an intervention can indeed result in significant improvements in school performance and also have a beneficial effect by reducing the number of individuals acquiring a criminal record at follow-up. They suggest that such early preventive intervention for those at risk of antisocial behaviour may benefit these individuals in the longer term.

\section{OPTIMALTREATMENTS FOR ANOREXIA AND GENETIC RISK IN SCHIZOPHRENIA}

There are limited contemporary high-quality data to guide the treatment of anorexia nervosa. Gowers et al (pp. 427-435) compared three treatment options: in-patient care, specialist out-patient treatment and generic treatment by the child and adolescent mental health team. The results show that all three groups improved over the 3 years of follow-up; however, only onethird of patients recovered fully. Contrary to their hypotheses, there were no significant differences between in-patient care and specialist out-patient care, when compared with routine treatment. They suggest that treatments administered without the patients' cooperation may be of limited value. An economic evaluation of the project demonstrated that the specialist out-patient option may be the most costeffective. There has been increased interest in the interaction between genetic vulnerability to developing schizophrenia and the contribution of cannabis and nicotine use. Zammit et al (pp. 402-407) found no association between two cannabinoid genes, or a nicotinic receptor gene, and schizophrenia or an interaction with drug use. Interestingly, they also failed to find a previously reported association between cannabis use and the catechol-O-methyltransferase $(C O M T)$ gene. They suggest that cannabis use is not associated with the ValMet COMT allele, and they do not find evidence of a gene-environment interaction between the COMT genotype and cannabis use exerting an effect on developing schizophrenia.

\section{PTSD AND TMS TREATMENT FOR DEPRESSION}

Post-traumatic stress disorder (PTSD) has been linked to disturbance of the hypothalamic-pituitary-adrenal axis. Meewise and colleagues (pp. 387-392) found that cortisol levels did not differ between patients with PTSD and healthy controls. However, they found lower levels of cortisol in those with PTSD who had been assessed in the afternoon, and lower levels in the control participants who had been exposed to trauma without developing PTSD. They suggest that the lower cortisol may be related to trauma exposure rather than PTSD per se. Repetitive transcranial magnetic stimulation (TMS) has been mooted as a treatment for depressive illness. Herwig et al (pp. 441-448) report the results of a study examining the efficacy of TMS as a strategy to augment standard antidepressant treatment. They found no differences in response rates between the TMS and control treatments.

\section{SUPERVISED TREATMENT, DUAL DIAGNOSIS AND STRUCTURED COMMUNICATION}

Compulsory community treatment has been proposed as a means of reducing the number of 'revolving door' admissions. Kisely \& Campbell (pp. 373-374) report that this has not occurred where such community treatment has been introduced in Victoria, Australia. They suggest that this policy may require further review before being introduced wholesale in the UK, as currently proposed in the new Mental Health Act. Dual diagnosis is a common yet difficult problem in clinical practice, particularly where psychosis and substance misuse coexist. Johnson et al (pp. 451-452) found that training case managers in managing dual diagnosis did not result in the predicted reduction in patient admissions or in their drug and alcohol consumption. They suggest that a different approach, perhaps using specialist staff, may be required in these cases. While regular meetings with patients are a standard part of psychiatric treatment, they vary considerably in their format. Priebe and colleagues (pp. 420-426) implemented a standardised computer-mediated intervention focused on systematically assessing patients' needs and their quality of life at each contact. Follow-up at 12 months demonstrated improvements in the patients' quality of life and the reporting of fewer unmet needs and greater treatment satisfaction. They suggest that this could be more widely implemented at relatively low cost. 